\title{
1 Single-cell Long Non-coding RNA Landscape of T Cells in Human 2 Cancer Immunity
}

3 Haitao Luo ${ }^{1,3,4, *, \#, \text { a }}$, Dechao Bu ${ }^{2, \#, b}$, Lijuan Shao ${ }^{1,3,4, \#, c}$, Yang Li $^{5, \mathrm{~d}}$, Liang Sun ${ }^{2, \mathrm{e}}$, Ce Wang ${ }^{1,3, \mathrm{f}}$,

4 Jing Wang ${ }^{1,3,4, \mathrm{~g}}$, Wei Yang ${ }^{1,3, \mathrm{~h}}$, Xiaofei Yang ${ }^{1,3, \mathrm{i}}$, Jun Dong ${ }^{4,{ }^{*}, \mathrm{j}}$, Yi Zhao ${ }^{2,{ }^{*}, \mathrm{k}}$ and Furong Li $^{1,3,{ }^{*}, 1}$

$5{ }^{1}$ Translational Medicine Collaborative Innovation Center, The Second Clinical Medical

6 College (Shenzhen People's Hospital), Jinan University, Shenzhen 518020, China

$7 \quad{ }^{2}$ Bioinformatics Research Group, Key Laboratory of Intelligent Information Processing,

8 Advanced Computing Research Center, Institute of Computing Technology, Chinese

9 Academy of Sciences, Beijing 100190, China

$10{ }^{3}$ Shenzhen key laboratory of stem cell research and clinical transformation, Shenzhen

11 518020, China

$12{ }^{4}$ Integrated Chinese and Western Medicine Postdoctoral research station, Jinan University,

13 Guangzhou 510632, China

$14{ }^{5}$ Department of Gastrointestinal Surgery, The Second Clinical Medical College (Shenzhen

15 People's Hospital), Jinan University, Shenzhen 518020, China

$17 *$ Corresponding authors.

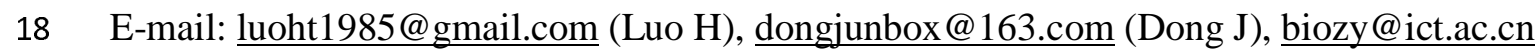

19 (Zhao Y), frli62@163.com (Li F).

$20 \quad$ Equal contribution.

21 Running title: Luo H et al / Single-cell LncRNA Landscape of T Cells

23 Total word counts (from "Introduction" to "Materials and methods"): 5,625

24 Total figures: 6

25 Total tables: 0

26 Total supplementary figures: 6

27 Total supplementary tables: 11 


\section{Abstract}

29 The development of new therapeutic targets for cancer immunotherapies and the 30 development of new biomarkers require deep understanding of $\mathrm{T}$ cells. To date, the complete 31 landscape and systematic characterization of long noncoding RNAs (lncRNAs) in T cells in 32 cancer immunity are lacking. Here, by systematically analyzing full-length single-cell RNA 33 sequencing (scRNA-seq) data of more than 20,000 T cell libraries across three cancer types, 34 we provide the first comprehensive catalog and the functional repertoires of IncRNAs in 35 human $\mathrm{T}$ cells. Specifically, we developed a custom pipeline for de novo transcriptome 36 assembly obtaining 9,433 novel lncRNA genes that increased the number of current human 37 lncRNA catalog by $16 \%$ and nearly doubled the number of lncRNAs expressed in T cells. We 38 found that a portion of expressed genes in single $\mathrm{T}$ cells were lncRNAs which have been 39 overlooked by the majority of previous studies. Based on metacell maps constructed by 40 MetaCell algorithm that partition scRNA-seq datasets into disjointed and homogenous groups 41 of cells (metacells), 154 signature lncRNAs associated with effector, exhausted, and 42 regulatory $\mathrm{T}$ cell states are identified, 84 of which are functionally annotated based on co43 expression network, indicating that lncRNAs might broadly participate in regulation of $\mathrm{T}$ cell 44 functions. Our findings provide a new point of view and resource for investigating the 45 mechanisms of $\mathrm{T}$ cell regulation in cancer immunity as well as for novel cancer-immune 46 biomarker development and cancer immunotherapies.

47 KEYWORDS: LncRNA; Transcriptome assembly; Metacell; Immune regulation; Functional 48 annotation 


\section{Introduction}

$51 \mathrm{~T}$ cell checkpoint inhibition therapies, such as targeting exhausted $\mathrm{CD}^{+} \mathrm{T}$ cells and 52 regulatory $\mathrm{T}$ cells (Tregs), have shown remarkable clinical benefit in many cancers [1-3].

53 Nevertheless, the mechanisms underlying therapy response or resistance are largely unknown, 54 which leads to the different therapeutic efficacies among cancer patients [4-8]. To better 55 understand the mechanisms that underlie successful response to immunotherapy, more 56 comprehensive studies to explore the whole transcriptome of individual $\mathrm{T}$ cells in tumor 57 ecosystems are desired. Long non-coding RNAs (lncRNAs), defined as a class of non-coding 58 RNAs longer than 200 nucleotides with no or low protein-coding potential, comprise a large 59 proportion of the mammalian transcriptome [9-12]. Accumulating evidence has suggested that lncRNAs are widely expressed in immune cells and play crucial roles in cancer immunity by regulating the differentiation and function of T cells [13-17]. For example, overexpression of $N K I L A$, an $N F-\kappa B$-interacting lncRNA, correlated with $\mathrm{T}$ cell apoptosis and shorter patient survival [18], and an enhancer-like lncRNA NeST regulates epigenetic marking patterns of $I F N$ - $\gamma$-encoding chromatin and induce synthesis of $I F N-\gamma$ in CD8 $\mathrm{T}$ cells [19]. However, previous studies seem to be somewhat scattered and the landscape and comprehensive functional analysis of lncRNAs in T cells in cancer immunity are still lacking.

The dramatic advances of single-cell RNA sequencing (scRNA-seq) technologies have gained unprecedented insight into the high diversity in $\mathrm{T}$ cell types and states compared to bulk RNA sequencing methods, which do not address the complex structures of tumor microenvironment [20-25]. Despite the advantages of single-cell resolution, in current most scRNA-seq studies of cancer immunology have generally focused on coding genes, overlooking the large amounts of lncRNAs. Detailed understanding of lncRNAs at the singlecell level was challenging owing to their relatively low and cell-specific expression [26-28]. As a widely used scRNA-seq approach, 3'-end sequencing technologies such as dropletbased 10X Genomics have lower RNA capture efficiencies, leading to the dropout evets and technological noise for lowly expressed lncRNAs [29]. Furthermore, accurate identification of novel lncRNAs is not suitable for the 3'-end sequencing technologies, but such analysis could be achieved by using full-length scRNA-seq technologies such as SMART-seq2 [30]. In addition, the sampling noise in scRNA-seq is generated through sampling of limited RNA transcripts from each cell [31], leading to a highly noisy estimation for most lncRNAs.

81 Therefore, to effectively characterize the lncRNA landscape at single-cell level, attention 82 should be paid to choosing appropriately scRNA-seq data and analytical approaches. 
83 Here, using unprecedentedly large-scale full-length single-cell transcriptome data of more 84 than 20,000 $\mathrm{T}$ cells from various tissues across three cancer types, we created a full annotation of the $\mathrm{T}$ cell lncRNA transcriptome and analyzed the functional roles associated with different $\mathrm{T}$ cell states. Our study aims to provide a basic and valuable resource for the future exploration of lncRNA regulatory mechanisms in $\mathrm{T}$ cells, which may facilitate novel cancer-immune biomarker development.

\section{Results}

\section{De novo transcriptome assembly of IncRNAs from scRNA-seq data of T cells}

To investigate the landscape of human lncRNAs in T cells across different tissues, patients and cancer types, we collected the data of 24,068 $\mathrm{T}$ cells (the size of the gzip-compressed FASTQ file was 7.5 TB) generated by full-length single-cell RNA sequencing with SMARTseq2, including the raw data of 9,878 cells from colorectal cancer (CRC) patients (2.8 TB), 10,188 cells from non-small-cell lung cancer (NSCLC) patients (3.1 TB), and 4,002 cells from 5 hepatocellular carcinoma (HCC) patients (1.6 TB) [32-34] (Figure S1A and Table S1). These cells were collected from peripheral blood, adjacent normal, and tumor tissue from each patient and sorted into $\mathrm{CD}^{+} \mathrm{CD}^{+}(\mathrm{CD} 8)$ and $\mathrm{CD}^{+} \mathrm{CD}^{+}(\mathrm{CD} 4) \mathrm{T}$ cells. The reads of each cell were mapped to the human reference genome (hg38/GRCh38), and the cells with unique mapping rates of less than $20 \%$ were removed. The remaining cells with on average 1.04 million uniquely mapped read pairs ( 0.63 million splices on average) and at least one pair of T cell receptor (TCR) $\alpha$ and $\beta$ chains enabled us to detect the expressed lncRNAs (Figure S1B-D).

Next, to generate the comprehensive $\mathrm{T}$ cell transcriptome beyond the currently reference annotation, we performed de novo transcriptome assembly using the StringTie method [35]. Although StringTie could be run by providing the reference annotation to guide the transcript construction, in current study we focused on to what extent it could assemble the whole transcriptome without the prior annotation. Based on the T cell dataset from HCC patients, we first measured the extent of assembly in each $\mathrm{T}$ cell and found that an average of 4,752 transcripts could be assembled at single-cell level, and an average of $69.8 \%(3,318 / 4,752)$ were matched to reference models (including reference protein-coding genes from 
To explore the best way to obtain novel transcripts, we compared the assembly results using three different approaches based on HCC dataset: (1) mapping and assembling for each single cell individually (cell-level); (2) assembling transcripts based on merged mapping results from each cell type of each patient (cell type-level); (3) assembling transcripts based on merged mapping results from each tissue of each patient (tissue-level). The transcripts assembled from each approach were merged independently and compared with reference genes respectively (Figure $\mathrm{S} 1 \mathrm{E}$ ). We found that the number of assembled transcripts matching to reference genes based on the cell type-level strategy (average 105,527 transcripts) was significantly higher than in cell-level or tissue-level methods (average 77,860 and 49,689 transcripts respectively, $P$-value $<0.001$, Wilcoxon rank sum test) (Figure 1B). Furthermore, the average number of matched transcripts from the cell type-level was more than twice that from the bulk-seq method (average 48,854 transcripts) (Figure 1B).

According to the cell-type pooling strategy, the cells from all patients across three cancer types were partitioned into 266 subsets (Figure 1C and Figure S1A), and the mapping results of cells from the same subset were merged and fed into assembling program. We found the number of assembled transcripts across different subsets showed positive correlations with the number of cells in these subsets in both CRC and NSCLC datasets (Pearson correlation coefficients $=0.6$ and $0.72, P$-value $=4.3 \mathrm{e}-11$ and $<2.2 \mathrm{e}-16$, respectively), but not in the HCC dataset (Pearson correlation coefficient $=0.22, P$-value $=0.17$ ) (Figure $1 \mathrm{D}$ and Figure $\mathrm{S} 1 \mathrm{~F})$. Then, assembled transcripts from all subsets were merged together, and a total of 751,710 primary genes were obtained. Next, we compared our assembled transcriptome with reference gene models. The results showed that reference lncRNAs had a lower detection rate than protein-coding genes. Specifically, $82 \%(16,399 / 19,938)$ of the known protein-coding genes in GENCODE v31 could be verified $(44 \%, 8,893 / 19,938$ were complete match with the same intron chain), while $16 \%(9,567 / 59,489)$ of known lncRNA genes were verified $(5 \%$, 3,140/59,489 were complete match) (Figure 1E). These findings suggested that lncRNAs were expressed in a much more cell-specific manner than protein-coding genes and further studies to uncover novel lncRNAs specifically expressed in human T cells were needed.

From the primary assembly, we developed a custom pipeline to identify novel lncRNAs. Briefly, we first selected transcripts that were no shorter than 200 nucleotides and have multiple exons. The transcripts that overlapped with both known protein-coding and known lncRNA genes were filtered out. Then, the transcripts lacking coding potential predicted by both CPC [36] and CNCI [37] utility were retained. Finally, the remaining transcripts that 
147 were reconstructed in at least two subsets with complete match were defined as the novel

148 IncRNA catalog (Figure 1C). Through this multi-layered analysis, we identified 9,433

149 previously unknown lncRNA genes (13,025 transcripts with mean length of 1,112

150 nucleotides), which increased the number of current human lncRNA catalog [38] by $16 \%$ and

151 nearly doubled the number of lncRNAs expressed in human T cells.

152 Finally, we performed experimental validation to evaluate the robustness of our identified 153 novel lncRNAs. First, fresh peripheral blood samples were collected from three CRC patients 154 (Table S2). Then, mononuclear cells were isolated from each sample. CD8 and CD4 T cells 155 were separated by immunomagnetic beads and the separation efficiency was verified by flow 156 cytometry (Figure 2A). Next, we selected 50 novel lncRNAs for quantitative real-time 157 polymerase chain reaction (qRT-PCR) analysis and Sanger sequencing across T cell samples. 158 As a result, 38 novel lncRNAs could be verified successfully by Sanger sequencing (Table 159 S3). As an example, for a novel lncRNA TCONS_00180551 located in an intergenic region of 160 chromosome 11, the blat search result of Sanger sequencing exactly matches the junction of 161 this novel lncRNA (Figure 2B).

\section{The characterization and expression analyses of IncRNAs in T cells}

Based on the relative genomic locations to reference protein-coding genes, the novel lncRNAs were classified into three locus biotypes, including 6,525 as intergenic, 3,187 as intronic and 3,313 as antisense lncRNAs. As in the case of reference lncRNAs, these novel lncRNAs showed fewer exons (the average number of exons was 2), lower detection rates and average gene abundance than protein-coding genes at single-cell level (Figure 3A-B). Specifically, by using pseudoalignment of scRNA-seq reads to both reference and novel lncRNA transcriptomes, on average 5,902 genes were detected (counts larger than 1) in each cell, $41 \%(2,397)$ of which were lncRNAs, including 1,258 reference and 1,139 novel lncRNAs (Figure 3A). Furthermore, for both reference and novel lncRNA genes, the average number of expressed genes across $\mathrm{T}$ cells was significantly lower than that of protein-coding genes. More precisely, we found that an average of 5,596 protein-coding and 2,093 lncRNA genes were expressed in at least $25 \%$ of cells. In such a situation, novel lncRNAs exhibited a higher average expression number and expression rate $(1,489$ and $15.8 \%, 1,489 / 9,433)$ than did reference lncRNAs (604 and 1\%, 604/59,489) (Figure 3B), suggesting that novel lncRNAs exhibited more enrichment than known lncRNAs in T cells in cancer. Moreover, we performed further analysis to investigate the specifically expressed lncRNAs in different 
tissues for each cancer type. In brief, for both CD4 and CD8 T cells of each cancer type, we identified 96 and 90 lncRNAs including 44 and 40 novel lncRNAs that expressed in tissuespecific pattern (Table S4). For example, some novel lncRNAs such as XLOC-301694 and $X L O C-126527$ were significantly expressed in CD4 $\mathrm{T}$ cells from tumor tissue of CRC (adjusted $P$ value $=3.17 \mathrm{E}-68$ and $1.72 \mathrm{E}-64$ respectively), while others such as XLOC-302096 and XLOC-502999 were significantly enriched in normal tissue and peripheral blood respectively (adjusted $P$ value $=9.18 \mathrm{E}-82$ and $1.35 \mathrm{E}-44$ respectively) (Table S4). Finally, we assessed the evolutionary conservation of these novel lncRNA transcripts and found that, on average, $61.2 \%$ have orthologous regions in the primate genomes, while only $3.4 \%$ mapped to mouse genome, suggesting the poor sequence conservation of these novel lncRNAs.

\section{Identification of signature IncRNAs associated with $T$ cell states in cancer immunity based on metacell maps}

To explore signature lncRNAs associated with $\mathrm{T}$ cell states in cancer immunity, we used the MetaCell method [31] that partitioned the scRNA-seq datasets into disjointed and homogeneous cell groups (namely metacells) using the non-parametric $K$-nn graph algorithm. For the lowly and specifically expressed nature of lncRNA genes, metacells pooling together data from cells derived from the same transcriptional states could serve as building blocks for approximating the distributions of lncRNA gene expression and minimizing the technical variance and noise. After quality control, 19,572 cells with predefined cluster annotations and 21,205 genes including both protein-coding and lncRNA genes were retained and used for the following analyses. The expression tables of CD8 and CD4 T cells across three cancers were fed into the MetaCell pipeline separately, resulting in a detailed map of 43 and 65 metacells respectively (Figure 4A-B and Table S5-6).

Based on the 2D projections (Figure 4A-B), predefined cell cluster annotations (Table $\mathrm{S} 1$ ), and the metacell similarity matrices (similarity among 43 or 65 metacells for CD8 or CD4 $\mathrm{T}$ cells respectively) (Figure S2A-B and Figure 4C-D), we organized the complex transcriptional landscape of CD8 into Naïve, effector/pre-effector, intermediated, and exhausted metacell groups and CD4 into Naïve, effector, intermediated, exhausted, and regulatory (including $\mathrm{FOXP} 3^{+} C T L A 4^{\text {low }}$ and $\mathrm{FOXP} 3^{+} C T L A 4^{\text {high }}$ ) metacell groups respectively (Figure 4C-D). To evaluate the composition of metacells, we mapped tissue- and cancerspecific patterns in all metacells and achieved results in accordance with previous studies [32-34] (Figure 4C-D and Figure S3-4). For example, exhausted metacells were preferentially 
211 enriched in tumors, while effector metacells were prevalent in peripheral blood. Although 212 some metacells were enriched in different cancer types, they were organized into the same 213 functional groups (Figure 4C-D). Notably, effector metacell groups (cytotoxic state) and 214 exhausted metacell groups (dysfunctional state) were located in different directions in the metacell maps, while the diffuse border was observed between the intermediate and the cytotoxic or dysfunctional state (Figure 4E-F). These intermediate cells exhibited remarkable transcriptional heterogeneity indicating functional divergence of these cells (Figure 4E-F and Figure S3-4). The observed cluster distribution in both CD8 and CD4 metacell maps might suggest a relative transition from activation to exhaustion that began with Naïve cells, followed by intermediate cells (such as central memory (CM), effector memory (EM) and tissue resident memory (RM) cells) and ended with exhausted cells. Moreover, the CD4 metacell map revealed that Tregs were subdivided into $F O X P 3^{+} C T L A 4^{\text {low }}$ Tregs and FOXP $3^{+} C T L A 4^{\text {high }}$ Tregs that were preferentially enriched in blood and tumors respectively (Figure 4D and 4F). These observations demonstrated that the diversity and dynamics of $\mathrm{T}$ cell states in cancer immune infiltrates could be controlled by complex and intricate gene regulatory mechanisms. Yet, the association between these cell states and lncRNAs was still poorly characterized, prompting us to subsequently investigate potential roles of lncRNA genes in T cells. Currently, the cell groups such as FOXP $3^{+} C T L A 4^{\text {high }}$ Tregs and exhausted $\mathrm{T}$ cells expressing inhibitory receptors (e.g., PDCDI and TIGIT) have been used as therapeutic targets for anti-cancer immunotherapies, thus we focused on these cells in the following analyses.

To explore signature lncRNAs associated with effector T cells, exhausted T cells, and Tregs, we performed systematic analysis of these metacell groups based on well-defined anchor genes [39], such as the genes associated with CD8 effector functions (CX3CRl, FGFBP2, GZMH and PRF1) or with the CD8 exhausted state (HAVCR2, LAG3, PDCD1, TIGIT and CTLA4). As a result, 154 lncRNAs that were significantly correlated to the anchor genes were identified and were involved in a set of co-expressed gene modules, including effector, exhausted and Treg gene modules (Figure 5A-B and Table S7). Interestingly, a putative $C T L A 4^{\text {high }}$ Treg gene subset was observed in the Treg module, suggesting its specific 240 functional roles in tumor-infiltrating Treg cells (Figure 5B). Overall, by combination analysis 241 with the expression profile across metacell groups, we found 47 and 79 lncRNAs correlated with effector and exhausted states in CD8 and CD4 cells respectively and were designated as 
244 were highly associated with Treg cells and were designated as Treg signature lncRNAs

245 (Figure S5). Among these signature lncRNAs, 14 and 7 lncRNA genes were shared between

246 CD8 and CD4 effector states and between CD8 and CD4 exhausted states respectively. 21

247 lncRNA genes associated with Tregs overlapped with those characteristics in the exhausted

248 CD4 T cells (Table S7), indicating the presence of shared regulatory roles of these lncRNAs.

249 In contrast, no signature lncRNA was shared between exhausted and effector states.

250 Functional prediction of signature IncRNAs associated with $\mathbf{T}$ cell states based on co251 expression network

252 To gain further insights into the functional roles of lncRNA in different $\mathrm{T}$ cell states in cancer, 253 we built a coding-noncoding network (CNC), as we previously reported [40, 41], using linear 254 correlation over all metacells. Applying this strategy, the functions of 54\% (84/154) signature 255 lncRNAs were annotated (Table S8). As expected, both CD8 and CD4 exhausted T cells have 256 the functional enrichments of signature lncRNAs that were markedly different from effector 257 CD8 or CD4 T cells, including regulation manners in immune system processes and several 258 signalling pathways (Figure 6A-B). For example, exhausted signature lncRNAs were 259 significantly enriched in immunoinhibitory functions such as negative regulation of immune 260 response (adjusted $P$-value $=2.96 \mathrm{e}-14)$, negative regulation of $\mathrm{T}$ cell activation $($ adjusted $P$ 261 value $=1.24 \mathrm{e}-06)$, and positive regulation of interleukin-10 biosynthetic process $($ adjusted $P$ 262 value $=1.02 \mathrm{e}-18$ ). In comparison, effector signature lncRNAs were enriched in cytotoxic 263 programs such as $\mathrm{T}$ cell proliferation involved in immune response (adjusted $P$-value $=$ 264 8.16e-09), positive regulation of cytokine secretion (adjusted $P$-value $=4.65 \mathrm{e}-05$ ), and 265 positive regulation of cytolysis (adjusted $P$-value $=1.59 \mathrm{e}-19)$ (Figure 6A-B and Table S9-10). 266 These results consisted with the phenotypes of exhausted or effector states of $\mathrm{T}$ cells as 267 described in previous studies [1, 32-34, 42]. In addition, the enriched functions of Treg 268 signature lncRNAs were similar with those of CD4 exhausted signature lncRNAs involving 269 multiple immunosuppressive programs (Figure 6C and Table S11), suggesting the shared 270 regulatory roles of these lncRNAs in CD4 Tregs and exhausted CD4 T cells. Further analysis 271 of the functions of co-signature lncRNAs that were shared between CD8 and CD4 exhausted 272 or effector states, as well as between CD4 exhausted and Treg states (Figure S6), suggests 273 that the signature lncRNAs might broadly participate in regulation of $\mathrm{T}$ cell functions within 274 the human tumor microenvironment. 
275 For example, a known lncRNA TM4SF19-AS1, defined as a signature lncRNA for both

276 CD8 effector and CD4 effector T cells and was transcribed in the antisense orientation to the

277 TM4SF19 gene, was co-expressed with 66 protein-coding and 11 lncRNA genes (Figure 6D-

278 E). Of note, TM4SF19-AS1 was highly correlated and located in the same topologically

279 associated domain (TAD) with its host gene TM4SF19 (Pearson correlation coefficient $=0.88$ )

280 (Figure 6D), a member of the four-transmembrane L6 superfamily participating in various

281 cellular processes including cell proliferation, motility, and cell adhesion [43-46].

282 Consistently, TM4SF19-AS1 was significantly enriched in several effector T cell associated

283 processes such as cellular response to cholesterol (adjusted $P$-value $=1.09 \mathrm{e}-30$ ), cell adhesion

284 (adjusted $P$-value $=5.25 \mathrm{e}-27$ ) and regulation of tumor necrosis factor biosynthetic process

285 (adjusted $P$-value $=3.75 \mathrm{e}-11)$ (Figure 6F). Interestingly, a recent study suggested that anti-

286 tumor response of CD8 T cells could be enhanced by regulating cholesterol metabolism [47].

287 For another example, a novel lncRNA XLOC-633950, defined as a signature lncRNA for

288 both CD4 exhausted T cells and Treg cells, was an intergenic gene and transcribed from the

289 promoter-enhancer cluster region of the SLA and CCN4 genes (Figure 6G). Furthermore,

290 XLOC-633950 as a novel gene, whose expression was supported by multiple expressed

291 sequence tags (EST), was located in the same TAD with the SLA gene which acted as an

292 inhibitor of antigen receptor signalling by negative regulation of positive selection and

293 mitosis of T cells [48-51] (Figure 6G). In accordance with SLA functions, the functional

294 enrichments of XLOC-633950 according to its co-expressed protein-coding genes were

295 mainly associated with immunoinhibitory processes, such as negative regulation of $\mathrm{T}$ cell

296 cytokine production (adjusted $P$-value $=4.56 \mathrm{e}-13$ ) and negative regulation of $\mathrm{T}$ cell

297 proliferation and activation (adjusted $P$-value $=7.25 \mathrm{e}-11$ and $5.85 \mathrm{e}-08$ respectively) (Figure

$2986 \mathrm{H}-\mathrm{I})$. These results provided a starting point for future dissecting the mechanisms of 299 signature lncRNAs.

\section{Discussion}

301 Despite the obvious advantages, most scRNA-seq data was still limited in its ability to study 302 IncRNAs, which were emerging as central players and key regulators in a number of 303 biological processes such as anti-tumor immune response [52, 53]. In comparison with many 304 scRNA-seq methods that amplified only the 3' end of transcripts, the SMART-seq2 protocol 305 could generate full-length cDNA from polyadenylated transcripts which results in data 306 suitable for analysis of lncRNAs [30, 54]. In current study, we preformed systematic analyses 
307 of SMART-seq2 full-length scRNA-seq datasets and provided the first comprehensive atlas of lncRNA in T cells of human cancer. containing 77,900 lncRNAs based on analysis of 14,166 polyA(+) RNA-Seq libraries and previous known annotations. Among the RefLnc lncRNAs, only 16\% could be assembled and expressed in $\mathrm{T}$ cells. In addition, compared with bulk-seq data, scRNA-seq data could detected more known and novel transcripts. These observations suggested that despite the vast number of lncRNAs that have been identified using bulk-seq data [10, 12, 26, 38, 55], the catalog of human lncRNAs is still far from being complete at single-cell resolution, due to their low and cell-specific expression patterns. Based on the cell-pooling strategy and more than 20,000 scRNA-seq libraries from 31 patients across three cancer types, we identified 9,433 previously non-annotated lncRNAs. These results significantly expand the current IncRNA catalog and enable us to carry out in-deep analysis of the $\mathrm{T}$ cell context-specific lncRNA transcriptome. Notably, all the scRNA-seq data used in current study was generated by sequencing the polyadenylated (ployA) transcriptome, in which non polyadenylated lncRNAs were absent.

Several previous studies have applied full-length scRNA-seq to unleash tumor infiltrating lymphocytes in HCC [34], NSCLC [32], and CRC[33], providing a deep understanding of the immune landscape of $\mathrm{T}$ cells in cancer. Nevertheless, the physiological function of lncRNAs in different $\mathrm{T}$ cell states during the cancer immune response remains elusive. Although the abundance of IncRNA was relatively low and hard to distinguish from technical noise in single $\mathrm{T}$ cells, pooling the transcripts from multiple cells that are derived from the same cell state allows more accurate quantification of lncRNAs, making it feasible to explore their signatures and putative regulatory mechanisms associated with $\mathrm{T}$ cell states in cancer immunity. Based on such partitioning and pooling strategies, we used the MetaCell method to identify homogeneous $\mathrm{T}$ cell groups from scRNA-seq data and derived a detailed map of 43 and 65 metacells for CD8 and CD4 T cells respectively. These metacells with higher homogeneity, allowed a more accurate quantification of lncRNAs as well as identification of $\mathrm{T}$ cell differentiation gradients. For example, we observed 7 metacells involved in CD8 effector cell cluster, which might reflect the transcriptional heterogeneity in this cluster (Figure 4C). The roles of lncRNAs in these different subsets (metacells) of CD8 effector T cells need further investigation. While MetaCell was not designed to perform single-cell 
339 lncRNA analysis, the MetaCell partitioning algorithm facilitated robust cell grouping of scRNA-seq data which enabled us to study lncRNAs more accurately.

According to the metacell maps (Figure 4E-F), in contrast to the pool of intermediate $\mathrm{T}$ cells with diffuse borders with other cell states, a discrete pool of effector T cells, exhausted

343 T cells and Tregs were observed that show clear gaps among them, thus facilitating unbiased 344 analysis of signature lncRNAs in these cell states. In total, the 154 signature lncRNAs were obtained providing a useful reference lncRNA resource to further investigate their functions in $\mathrm{T}$ cell mediated cancer immunity. Since lncRNAs generally interact with protein-coding genes, and highly correlated genes generally have similar functions, the putative functions of these signature lncRNAs could be predicted by the co-expressed coding genes. Therefore, by constructing the 'two color' co-expression network in which both coding and lncRNA genes were involved, the functions of 84 signature lncRNAs were annotated. Some lncRNAs were genomically co-located with their host genes, that revealed the complicated regulation mechanisms of lncRNAs in cancer immunity. For example, as described above, TM4SF19AS1 was both co-expressed and co-located with their host gene TM4SF19, whose family has functions in various biological processes including cell proliferation and adhesion that are consistent with the characteristics of effector T cells [43-46].

In summary, the current study provides the first comprehensive catalog and the functional repertoires of lncRNAs in human cancer T cells. Although the expression pattern and exact mechanisms of these signature lncRNAs in regulating $\mathrm{T}$ cell states needs further experimental validation, we provide the groundwork for future studies to investigate the functional mechanisms of lncRNAs in the $\mathrm{T}$ cell mediated cancer immunity, especially in two of the essential states of $\mathrm{T}$ cells: effector state and exhausted state. These signature lncRNAs of CD8 exhausted $\mathrm{T}$ cells and tumor Tregs, may serve as new targets for novel cancer-immune biomarker development and cancer immunotherapies.

\section{Materials and methods}

\section{Full-length scRNA-seq and bulk RNA-seq datasets from cancer patients}

Raw sequencing data of three compendium datasets used in the current study were authorized by the European Genome-phenome Archive (EGA) and obtained from the EGA database under study accession id: EGAS00001002791, EGAS00001002430, and EGAS00001002072. 
370 cells isolated from different tissues (peripheral blood, adjacent normal and tumor tissues) of

37112 CRC patients [33]. The NSCLC scRNA-seq dataset (EGAS00001002430) contains the 372 raw data of 12,346 single T cells from 14 NSCLC patients [32]. The HCC scRNA-seq dataset 373 (EGAS00001002072) contains the raw data of 5,063 single T cells from 6 HCC patients [34]. 374 All the data were generated by Illumina HiSeq 2500 sequencer with 100 bp pair-end reads or 375 Illumina Hiseq 4000 sequencer with 150 bp pair-end reads. The cells from HCC patient 376 P1202 (TCRs could not be assembled in those cells) were not analyzed in the current study. 377 After preliminary filtration, 24,075 T cells with at least one pair of TCR alpha-beta chain 378 were retained. The bulk RNA-seq data of five tumor samples from HCC patients were obtained from HCC dataset.

According to the cell annotations from original papers [32-34], these $\mathrm{T}$ cells were classified into different subtypes (Figure S1A and Table S1). PTC, NTC, and TTC represent $\mathrm{CD}^{+} \mathrm{CD}^{+} \mathrm{T}$ cells that were isolated from peripheral blood, adjacent normal, and tumor tissues respectively. The PTH, NTH, and TTH represent $\mathrm{CD} 3{ }^{+} \mathrm{CD} 4{ }^{+} \mathrm{CD} 25^{\text {low }} \mathrm{T}$ cells that were isolated from the three tissues. PTR, NTR, and TTR represent $\mathrm{CD} 3^{+} \mathrm{CD} 4{ }^{+} \mathrm{CD} 25^{\text {high }} \mathrm{T}$ cells that were isolated from the three tissues.

\section{Reads mapping and transcripts assembly}

Clean reads from each $\mathrm{T}$ cell were mapped to the human reference genome (version hg38/GRCh38) using STAR aligner (v2.7.1) [56] with the twopassMode set as Basic. The bam files of $\mathrm{T}$ cells from each cell-type of each patient were merged using SAMtools merge [57]. StringTie (v2.0.3) [35] was used to assemble transcripts based on genomic read alignments. Assembled transcripts of all cell-types across all patients were merged together using the Cuffmerge utility of Cufflinks package [58].

\section{Comparison with reference gene annotation}

For reference gene annotation, lncRNA genes were collected from RefLnc [38] and other genes were collected from GENCODE v31 [59]. According to the "class code" information outputted by Cuffcompare, the merged assembly was classified into four categories by comparison with the reference gene annotation, including known coding genes, known lncRNA genes, potentially novel genes (class code is " $\mathrm{i}, \mathrm{x}, \mathrm{u}$ "), and others. 
400 Based on the potentially novel gene catalog derived from single-cell data, we developed a

401 custom pipeline for identification of reliable novel lncRNAs including the following steps: (1)

402 transcripts that are no shorter than $200 \mathrm{nt}$ and have more than one exon were selected for 403 downstream analysis (for intergenic transcripts, at least $1 \mathrm{~kb}$ away from known protein404 coding genes); (2) CPC (Coding Potential Calculator) [36] and CNCI (Coding Noncoding 405 Index) [37] software were used to evaluate the protein-coding potential of transcripts, and 406 transcripts that were reported to lack coding potential by both CPC and CNCI were regarded 407 as candidate noncoding transcripts; (3) The remaining transcripts that were assembled and 408 have the same intron chain of at least two cell-types were retained as the final novel lncRNA 409 catalog. The final lncRNA catalog was obtained by combining the reference lncRNA and 410 novel lncRNA genes directly. The UCSC liftOver tool (http://genome.ucsc.edu/cgi411 bin/hgLiftOver?hgsid=806106955 h2xhcK2iPRI7SiMkxkB41I2mwF9O) was used to 412 identify the orthologous locations of human novel lncRNAs in the mouse genome and in 413 primates such as chimpanzee and gorilla, with the parameters: Minimum ratio of bases that 414 must remap $=0.1$ and Min ratio of alignment blocks or exons that must map $=0.5$.

\section{Experimental validation of novel lncRNAs}

416 Three CRC patients were enrolled at Shenzhen People's Hospital. The informed consent 417 forms were provided by patients. The current study was approved by Medical Ethics 418 Committee of Shenzhen People's Hospital. The clinical characteristics of three patients are 419 summarized in Table S2. Peripheral blood samples from three patients were obtained and 420 treated with anticoagulation. Peripheral blood mononuclear cells (PBMCs) were extracted by 421 Ficoll-Paque Plus (GE Healthcare, Sweden, 17144003). Then, $\mathrm{CD}^{+}$and $\mathrm{CD}^{+} \mathrm{T}^{\mathrm{T}}$ cells were 422 separated by immunomagnetic beads (Meltenyi Biotec, Germany, 130045101, 130045101). 423 The separation efficiency was verified by flow cytometry. The sorted cells were dissolved in 424 Trizol Reagent (Ambion, USA, 15596026) for RNA extraction according to the 425 manufacture's protocol. cDNA was synthesized by PrimerScript RT reagent kit (Takara, 426 Japan, AHG1552A). We chose 50 novel lncRNAs to perform experimental validation 427 according to the following criteria: (1) highly expressed in either CD8 or CD4 T cells; (2) 428 reconstructed in at least ten subsets with complete match; (3) uniquely mapped to human 429 genome. For each lncRNA, at least two pairs of primers for qRT-PCR were designed using 430 NCBI Primer-BLAST (https://www.ncbi.nlm.nih.gov/tools/primer-blast). In order to ensure 431 the specificity of primers, UCSC InSilicon PCR (http://genome.ucsc.edu/cgi-bin/hgPcr) was 432 used to compare the primer pairs with human genome (hg38). Some primer pairs were 
433 specifically designed to span splicing sites (exon junctions). QRT-PCR were performed with 434 SYBR Green master mix (Takara, Japan) on an ABI StepOnePlus (Applied Biosystems, 435 USA). GAPDH as housekeeping gene was used as positive control. For each lncRNA, we 436 selected one primer pair product of qRT-PCR for Sanger sequencing.

\section{Quality control (QC) and normalization}

438 We calculated the read counts and transcripts per million (TPM) values using 439 pseudoalignment of scRNA-seq reads to both protein-coding and lncRNA transcriptomes, as 440 implemented in Kallisto (v0.46.0) [60] with default parameters, and summarized expression 441 levels from the transcript level to the gene level.

442 Low-quality and doublet cells were removed if the number of expressed genes (counts of 443 more than 1) was fewer than 2000 or higher than the medians of all cells plus $3 \times$ the median 444 absolute deviation, respectively. Moreover, the cells with the proportion of reads mapped to 445 mitochondrial genes was larger than $10 \%$ were discarded. Genes with average counts of more 446 than 1 and expressed in at least $1 \%$ of cells for each type of cancer were retained. The 447 combined count tables from all $\mathrm{T}$ cells passing the above filtration were normalized using a 448 pooling and deconvolution method implemented in the $R$ package named 449 computeSumFactors [61] with the sizes ranged from 80, 100, 120 to 140. According to the 450 assumption that most genes were not differentially expressed, normalization was performed 451 within each predefined cluster separately to compute cell size factors. The cell size factors 452 were rescaled by normalization among clusters. Finally, the counts for each cell were 453 normalized by dividing the cell counts by the cell size factor.

\section{$454 \quad$ MetaCell modeling}

455 The MetaCell method [31], that partitioned the scRNA-seq dataset into disjointed and 456 homogeneous cell groups (metacells) using the $K$-nn graph algorithm, was performed for 457 both the CD8 and CD4 T cells independently. We first removed specific mitochondrial genes 458 (annotated with the prefix "MT-"), that typically mark cells as being stressed or dying, rather 459 than cellular identity. Based on the count matrices of both protein-coding and lncRNA genes, 460 feature genes whose scaled variance (variance/mean on down-sampled matrices) exceeded 4610.08 were selected and used to compute cell-to-cell similarity using Pearson correlations. 462 According to the cell-to-cell similarity matrices, two balanced $K$-nn similarity graphs for 463 CD8 and CD4 T cells were constructed using the parameter $K=100$ (the number of neighbors 
464 for each cell was limited by $K$ ). Next, we performed the resampling procedures (resampling $46575 \%$ of the cells in each iteration with 500 iterations) and co-clustering graph construction 466 (the minimal cluster size was 50). Finally, the graphs of metacells (and the cells belonging to 467 them) were projected into 2D spaces to explore the similarities between cells and metacells.

\section{$468 \quad$ Annotation of metacells}

469 Annotation of metacells was performed based on the metacell confusion matrix and 470 predefined cluster annotations (File S1) of T cells involved in the metacells. Briefly, we first 471 created a hierarchical clustering of metacells according to the number of similarity 472 relationships between their cells. Next, we generated clusters of metacells as confusion 473 matrices based on the hierarchy results, then annotated these clusters according to the 474 annotations of $\mathrm{T}$ cells.

\section{Defining signature IncRNAs associated with $\mathbf{T}$ cell states}

476 To identify signature lncRNAs associated with effector and exhausted $\mathrm{T}$ cells as well as 477 Tregs, as described in recent study [39], we adopted the anchor approach by identifying the 478 lncRNAs that were significantly correlated to well-defined anchor genes, based on metacells' $479 \log$ enrichment scores (lfp values calculated by MetaCell method). The lncRNAs that 480 significantly correlated with anchor genes (adjusted $P$-value $<0.01$ and ranked in the top 0.05 481 percentile for each anchor gene) were regarded as signature lncRNAs. The anchor genes were defined as follows: the anchor genes of CD8 exhausted T cells included HAVCR2, LAG3, PDCD1, TIGIT, and CTLA4; the anchor genes of CD8 effector T cells included CX3CRI, FGFBP2, GZMH and PRF1; genes associated with Tregs included FOXP3; the anchor genes of CD4 exhausted T cells included CXCL13, PDCD1, HAVCR2, TIGIT, and CTLA4; genes associated with CD4 effector T cells included GNLY, GZMB, GZMH, PRF1, and NKG7.

\section{Function prediction of signature IncRNAs based on co-expression network}

Based on lfp values of both lncRNA and protein-coding genes across all metacells, we used a custom pipeline for large-scale prediction of signature lncRNA functions by constructing the coding-lncRNA gene co-expression network [40,41]. Briefly, genes with log enrichment scores ranked in the top $75 \%$ of each metacell were retained. Then, $P$-values of Pearson correlation coefficients for each gene pair were calculated based on the Fisher's asymptotic test using the WGCNA package of R. $P$-values were adjusted based on the Bonferroni multiple test correction using the multtest package of R. The gene pairs with an adjusted $P$ - 
495 value $<0.01$, Pearson correlation coefficient $>0.7$, and ranked in the top 5\% for each gene 496 were involved in co-expression network.

497 Based on the co-expression network, lncRNA functions were predicted using module498 and hub-based methods. Specifically, the Markov cluster algorithm was adopted to identify 499 co-expressed modules [40]. For each module, if the known genes were significantly enriched 500 for at least one Gene Ontology (GO) term, the functions of the lncRNAs involved in the 501 module were assigned as the same ones. For hub-based method, the functions of a hub 502 lncRNA (node degree > 10) were assigned, if its immediate neighboring genes were 503 significantly enriched for at least one GO term.

\section{Data availability}

505 All the novel lncRNA genes identified in current study and their expression files are available 506 in the NONCODE database (http://www.noncode.org/download.php).

\section{Authors' contributions}

508 HL, DB, JD, YZ and FL conceptualized and designed the study. HL and DB led the data 509 analysis. HL performed the study and interpreted data. LJS performed experimental 510 validation. YL collected the clinical samples and prepared the experimental materials. LS 511 optimized the CNCI algorithm. WY, CW, XY and JW collected the data and performed T 512 cell annotations. HL wrote the manuscript. HL, YZ and FL revised the manuscript. JD, YZ 513 and FL supervised the project. All authors read and approved the final manuscript.

\section{Competing interests}

515 The authors have declared no competing interests.

\section{Acknowledgments}

517 This work was supported by the Science and Technology Project of Shenzhen (No. 518 JHZ20170310090257380, JCYJ20170413092711058, JCYJ20170307095822325), China 519 Postdoctoral Science Foundation (No. 2019M663369), Natural Science Foundation of 520 Shenzhen (20190727160324164), and National Natural Science Foundation of China 521 (31970636). We thank Dr. Lei Zhang and Yao He at Peking University for assistance with 
522 raw data download. The data analysis was performed on AWS (China region) and we thank

523 Mr Hansen Huang, Chao Wu and Fei Shi for providing the technical service and support.

\section{Authors' ORCID IDs}

525 a 0000-0003-3671-7786 (Luo, H)

$526 \quad{ }^{\mathrm{b}} 0000-0002-8833-5432(\mathrm{Bu}, \mathrm{D})$

$527 \quad$ C0000-0001-7980-2003 (Shao, L)

$528 \quad{ }^{\mathrm{d}} 0000-0001-8199-8527(\mathrm{Li}, \mathrm{Y})$

$529 \quad{ }^{\mathrm{e}} 0000-0002-5213-6941$ (Sun, L)

530 f 0000-0003-3048-7596 (Wang, C)

531 g0000-0001-7856-4533 (Wang, J)

$532{ }^{\mathrm{h}}$ 0000-0003-2138-5563 (Yang, W)

533 i0000-0001-6255-5181 (Yang, X)

534 j0000-0003-4064-3134 (Dong, J)

$535 \quad{ }^{k}$ 0000-0001-6046-8420 (Zhao, Y)

$536 \quad{ }^{\mathrm{l}} 0000-0002-0606-8861(\mathrm{Li}, \mathrm{F})$

\section{References}

539 [1] Chen DS, Mellman I. Elements of cancer immunity and the cancer-immune set point.

$540 \quad$ Nature 2017;541:321-30.

541 [2] Bodor JN, Boumber Y, Borghaei H. Biomarkers for immune checkpoint inhibition in non542 small cell lung cancer (NSCLC). Cancer 2020;126:260-70.

543 [3] Houot R, Schultz LM, Marabelle A, Kohrt H. T-cell-based immunotherapy: adoptive cell 544 transfer and checkpoint inhibition. Cancer Immunol Res 2015;3:1115-22. 
545 [4] Restifo NP, Dudley ME, Rosenberg SA. Adoptive immunotherapy for cancer: harnessing

546 the T cell response. Nat Rev Immunol 2012;12:269-81.

547 [5] Hilmi M, Vienot A, Rousseau B, Neuzillet C. Immune therapy for liver cancers. Cancers 548 (Basel) 2019;12.

549 [6] Carter JA, Gilbo P, Atwal GS. IMPRES does not reproducibly predict response to 550 immune checkpoint blockade therapy in metastatic melanoma. Nat Med 2019;25:1833-5.

551 [7] Hugo W, Zaretsky JM, Sun L, Song C, Moreno BH, Hu-Lieskovan S, et al. Genomic and 552 transcriptomic features of response to anti- $P D-1$ therapy in metastatic melanoma. Cell 553 2016;165:35-44.

554 [8] Auslander N, Zhang G, Lee JS, Frederick DT, Miao B, Moll T, et al. Robust prediction of 555 response to immune checkpoint blockade therapy in metastatic melanoma. Nat Med $556 \quad 2018 ; 24: 1545-9$.

557 [9] Rinn JL, Chang HY. Genome regulation by long noncoding RNAs. Annu Rev Biochem 558 2012;81:145-66.

559 [10] Fang S, Zhang L, Guo J, Niu Y, Wu Y, Li H, et al. NONCODEV5: a comprehensive 560 annotation database for long non-coding RNAs. Nucleic Acids Res 2018;46:D308-D14.

561 [11] Harrow J, Frankish A, Gonzalez JM, Tapanari E, Diekhans M, Kokocinski F, et al. 562 GENCODE: the reference human genome annotation for The ENCODE Project. Genome Res $563 \quad 2012 ; 22: 1760-74$.

564 [12] Iyer MK, Niknafs YS, Malik R, Singhal U, Sahu A, Hosono Y, et al. The landscape of 565 long noncoding RNAs in the human transcriptome. Nat Genet 2015;47:199-208.

566 [13] Yu WD, Wang H, He QF, Xu Y, Wang XC. Long noncoding RNAs in cancer-immunity 567 cycle. J Cell Physiol 2018;233:6518-23.

568 [14] Wang L, Felts SJ, Van Keulen VP, Scheid AD, Block MS, Markovic SN, et al. 569 Integrative genome-wide analysis of long noncoding RNAs in diverse immune cell types of 570 melanoma patients. Cancer Res 2018;78:4411-23.

571 [15] Xu J, Cao X. Long noncoding RNAs in the metabolic control of inflammation and 572 immune disorders. Cell Mol Immunol 2019;16:1-5.

573 [16] Spurlock CF, Crooke PS, Aune TM. Biogenesis and transcriptional regulation of long 574 noncoding RNAs in the human immune system. J Immunol 2016;197:4509-17.

575 [17] Fanucchi S, Fok ET, Dalla E, Shibayama Y, Borner K, Chang EY, et al. Immune genes 576 are primed for robust transcription by proximal long noncoding RNAs located in nuclear 577 compartments. Nat Genet 2019;51:138-50. 
578 [18] Huang D, Chen J, Yang L, Ouyang Q, Li J, Lao L, et al. NKILA lncRNA promotes 579 tumor immune evasion by sensitizing T cells to activation-induced cell death. Nat Immunol $580 \quad 2018 ; 19: 1112-25$.

581 [19] Gomez JA, Wapinski OL, Yang YW, Bureau JF, Gopinath S, Monack DM, et al. The 582 NeST long ncRNA controls microbial susceptibility and epigenetic activation of the 583 interferon-gamma locus. Cell 2013;152:743-54.

584 [20] Ren X, Kang B, Zhang Z. Understanding tumor ecosystems by single-cell sequencing: 585 promises and limitations. Genome Biol 2018;19:211.

586 [21] Chung W, Eum HH, Lee HO, Lee KM, Lee HB, Kim KT, et al. Single-cell RNA-seq 587 enables comprehensive tumour and immune cell profiling in primary breast cancer. Nat 588 Commun 2017;8:15081.

589 [22] Lavin Y, Kobayashi S, Leader A, Amir ED, Elefant N, Bigenwald C, et al. Innate 590 immune landscape in early lung adenocarcinoma by paired single-cell analyses. Cell $591 \quad 2017 ; 169: 750-65$ e17.

592 [23] Zhang Q, He Y, Luo N, Patel SJ, Han Y, Gao R, et al. Landscape and dynamics of single 593 immune cells in hepatocellular carcinoma. Cell 2019;179:829-45 e20.

594 [24] Savas P, Virassamy B, Ye C, Salim A, Mintoff CP, Caramia F, et al. Single-cell 595 profiling of breast cancer $\mathrm{T}$ cells reveals a tissue-resident memory subset associated with 596 improved prognosis. Nat Med 2018;24:986-93.

597 [25] Azizi E, Carr AJ, Plitas G, Cornish AE, Konopacki C, Prabhakaran S, et al. Single-cell 598 map of diverse immune phenotypes in the breast tumor microenvironment. Cell 599 2018;174:1293-308 e36.

600 [26] Uszczynska-Ratajczak B, Lagarde J, Frankish A, Guigo R, Johnson R. Towards a 601 complete map of the human long non-coding RNA transcriptome. Nat Rev Genet $602 \quad 2018 ; 19: 535-48$.

603 [27] Stegle O, Teichmann SA, Marioni JC. Computational and analytical challenges in 604 single-cell transcriptomics. Nat Rev Genet 2015;16:133-45.

605 [28] Vallejos CA, Risso D, Scialdone A, Dudoit S, Marioni JC. Normalizing single-cell RNA 606 sequencing data: challenges and opportunities. Nat Methods 2017;14:565-71.

607 [29] Zheng GX, Terry JM, Belgrader P, Ryvkin P, Bent ZW, Wilson R, et al. Massively 608 parallel digital transcriptional profiling of single cells. Nat Commun 2017;8:14049.

609 [30] Picelli S, Faridani OR, Bjorklund AK, Winberg G, Sagasser S, Sandberg R. Full-length 610 RNA-seq from single cells using Smart-seq2. Nat Protoc 2014;9:171-81. 
611 [31] Baran Y, Bercovich A, Sebe-Pedros A, Lubling Y, Giladi A, Chomsky E, et al. MetaCell: 612 analysis of single-cell RNA-seq data using K-nn graph partitions. Genome Biol 2019;20:206.

613 [32] Guo X, Zhang Y, Zheng L, Zheng C, Song J, Zhang Q, et al. Global characterization of 614 T cells in non-small-cell lung cancer by single-cell sequencing. Nat Med 2018;24:978-85.

615 [33] Zhang L, Yu X, Zheng L, Zhang Y, Li Y, Fang Q, et al. Lineage tracking reveals 616 dynamic relationships of T cells in colorectal cancer. Nature 2018;564:268-72.

617 [34] Zheng C, Zheng L, Yoo JK, Guo H, Zhang Y, Guo X, et al. Landscape of infiltrating T 618 cells in liver cancer revealed by single-cell sequencing. Cell 2017;169:1342-56 e16.

619 [35] Pertea M, Pertea GM, Antonescu CM, Chang TC, Mendell JT, Salzberg SL. StringTie 620 enables improved reconstruction of a transcriptome from RNA-seq reads. Nat Biotechnol $621 \quad 2015 ; 33: 290-5$.

622 [36] Kong L, Zhang Y, Ye ZQ, Liu XQ, Zhao SQ, Wei L, et al. CPC: assess the protein623 coding potential of transcripts using sequence features and support vector machine. Nucleic 624 Acids Res 2007;35:W345-9.

625 [37] Sun L, Luo H, Bu D, Zhao G, Yu K, Zhang C, et al. Utilizing sequence intrinsic 626 composition to classify protein-coding and long non-coding transcripts. Nucleic Acids Res $627 \quad 2013 ; 41: \mathrm{e} 166$.

628 [38] Jiang S, Cheng SJ, Ren LC, Wang Q, Kang YJ, Ding Y, et al. An expanded landscape of human long noncoding RNA. Nucleic Acids Res 2019;47:7842-56. [39] Li H, van der Leun AM, Yofe I, Lubling Y, Gelbard-Solodkin D, van Akkooi ACJ, et al. Dysfunctional CD8 T cells form a proliferative, dynamically regulated compartment within human melanoma. Cell 2019;176:775-89 e18.

[40] Liao Q, Liu C, Yuan X, Kang S, Miao R, Xiao H, et al. Large-scale prediction of long non-coding RNA functions in a coding-non-coding gene co-expression network. Nucleic 635 Acids Res 2011;39:3864-78.

636 [41] Luo H, Bu D, Sun L, Fang S, Liu Z, Zhao Y. Identification and function annotation of 637 long intervening noncoding RNAs. Brief Bioinform 2017;18:789-97.

638 [42] Wherry EJ, Kurachi M. Molecular and cellular insights into T cell exhaustion. Nat Rev 639 Immunol 2015;15:486-99.

640 [43] Wright MD, Ni J, Rudy GB. The $L 6$ membrane proteins--a new four-transmembrane 641 superfamily. Protein Sci 2000;9:1594-600.

642 [44] Chang YW, Chen SC, Cheng EC, Ko YP, Lin YC, Kao YR, et al. CD13 643 (aminopeptidase N) can associate with tumor-associated antigen $L 6$ and enhance the motility 644 of human lung cancer cells. Int J Cancer 2005;116:243-52. 
645 [45] Lekishvili T, Fromm E, Mujoomdar M, Berditchevski F. The tumour-associated antigen

$646 L 6(L 6-A g)$ is recruited to the tetraspanin-enriched microdomains: implication for tumour cell 647 motility. J Cell Sci 2008;121:685-94.

648 [46] Allioli N, Vincent S, Vlaeminck-Guillem V, Decaussin-Petrucci M, Ragage F, Ruffion

649 A, et al. TM4SF1, a novel primary androgen receptor target gene over-expressed in human 650 prostate cancer and involved in cell migration. Prostate 2011;71:1239-50.

651 [47] Yang W, Bai Y, Xiong Y, Zhang J, Chen S, Zheng X, et al. Potentiating the antitumour 652 response of CD8(+) T cells by modulating cholesterol metabolism. Nature 2016;531:651-5.

653 [48] Marton N, Baricza E, Ersek B, Buzas EI, Nagy G. The Emerging and diverse roles of 654 Src-like adaptor proteins in health and disease. Mediators Inflamm 2015;2015:952536.

655 [49] Sosinowski T, Pandey A, Dixit VM, Weiss A. Src-like adaptor protein (SLAP) is a 656 negative regulator of T cell receptor signaling. J Exp Med 2000;191:463-74.

657 [50] Pandey A, Ibarrola N, Kratchmarova I, Fernandez MM, Constantinescu SN, Ohara O, et 658 al. A novel $S r c$ homology 2 domain-containing molecule, $S r c$-like adapter protein-2 (SLAP-2), 659 which negatively regulates T cell receptor signaling. J Biol Chem 2002;277:19131-8.

660 [51] Park SK, Beaven MA. Mechanism of upregulation of the inhibitory regulator, src-like 661 adaptor protein (SLAP), by glucocorticoids in mast cells. Mol Immunol 2009;46:492-7.

662 [52] Yao RW, Wang Y, Chen LL. Cellular functions of long noncoding RNAs. Nat Cell Biol $6632019 ; 21: 542-51$.

664 [53] Agirre X, Meydan C, Jiang Y, Garate L, Doane AS, Li Z, et al. Long non-coding RNAs 665 discriminate the stages and gene regulatory states of human humoral immune response. Nat 666 Commun 2019;10:821.

667 [54] Liu F, Zhang Y, Zhang L, Li Z, Fang Q, Gao R, et al. Systematic comparative analysis 668 of single-nucleotide variant detection methods from single-cell RNA sequencing data. 669 Genome Biol 2019;20:242.

670 [55] Quek XC, Thomson DW, Maag JL, Bartonicek N, Signal B, Clark MB, et al. IncRNAdb 671 v2.0: expanding the reference database for functional long noncoding RNAs. Nucleic Acids 672 Res 2015;43:D168-73.

673 [56] Dobin A, Davis CA, Schlesinger F, Drenkow J, Zaleski C, Jha S, et al. STAR: ultrafast 674 universal RNA-seq aligner. Bioinformatics 2013;29:15-21.

675 [57] Li H, Handsaker B, Wysoker A, Fennell T, Ruan J, Homer N, et al. The sequence 676 alignment/map format and SAMtools. Bioinformatics 2009;25:2078-9. 
677 [58] Trapnell C, Williams BA, Pertea G, Mortazavi A, Kwan G, van Baren MJ, et al.

678 Transcript assembly and quantification by RNA-Seq reveals unannotated transcripts and 679 isoform switching during cell differentiation. Nat Biotechnol 2010;28:511-5.

680 [59] Frankish A, Diekhans M, Ferreira AM, Johnson R, Jungreis I, Loveland J, et al. 681 GENCODE reference annotation for the human and mouse genomes. Nucleic Acids Res 682 2019;47:D766-D73.

683 [60] Bray NL, Pimentel H, Melsted P, Pachter L. Near-optimal probabilistic RNA-seq 684 quantification. Nat Biotechnol 2016;34:525-7.

685 [61] Lun AT, Bach K, Marioni JC. Pooling across cells to normalize single-cell RNA 686 sequencing data with many zero counts. Genome Biol 2016;17:75.

\section{Figure Legends}

689 Figure 1 The statistics of assembled transcripts and workflow for novel IncRNA 690 identification process in $\mathbf{T}$ cells during cancer immunity

691 A. Violin plots showing the number of assembled transcripts and the number of those matched to the reference at single cell level across five HCC patients. B. Number of assembled transcripts that matched to reference across five HCC patients based on four different strategies. *** indicates $P$-value $<0.001$ (Wilcoxon rank sum test). C. Correlation of the number of cells and the number of assembled transcripts across different subsets for CRC, HCC and NSCLC. A 95\% confidence interval was added and shown as coloured regions. D. Scheme of pipeline used to identify the novel lncRNAs expressed in $\mathrm{T}$ cells during cancer immunity using three full-length scRNA-seq datasets. E. The statistics of assembled transcripts that matched to reference protein-coding and reference lncRNA genes. CRC, colorectal cancer; HCC, hepatocellular carcinoma; NSCLC, non-small-cell lung cancer; $\mathrm{P}$, peripheral blood; $\mathrm{N}$, adjacent normal tissue; $\mathrm{T}$, tumor tissue.

Figure 2 Single $T$ cell sorting and quality evaluation of an example novel lncRNA

A. The results of flow cytometric analysis. CD8 and CD4 T cells from three patients were separated by magnetic beads and stained with flow cytometry antibody CD8-APC and CD4APC respectively (Isotype was used as negative control). B. An example of novel intergenic 706 IncRNA that was validated by Sanger sequencing. The genomic views are generated from UCSC genome browser. The spliced sequence outputted by Sanger sequencing is shown. 
708 Figure 3 Characterization of IncRNA expression patterns at single-cell level

709 A. The number of protein-coding, reference lncRNA, and novel lncRNA genes expressed in

710 T cells across three cancer types. *** indicates $P$-value $<0.001$ (Wilcoxon rank sum test). B.

711 The plots show the percentage of expressing cells against the mean expression level

712 (logCounts) for protein-coding, reference lncRNA, and novel lncRNA genes across three

713 cancer types. The numbers of genes that are expressed in at least $25 \%$ of cells are labelled.

714 Figure 4 Characterization of $T$ cell states based on $2 D$ projection of $T$ cells and the 715 annotation of metacell maps

716 A. 2D projection of CD8 T cells from three cancer types into 43 metacells. B. 2D projection 717 of CD4 T cells from three cancer types into 65 metacells. C, D. CD8 (C) and CD4 (D) 718 metacells (rows) are ordered by groups and organized within each group. The first panel of 719 the bar plot shows the number of cells of different clusters in each metacell. The second and 720 third panel of the bar plots show the percentage of cells from different cancer types and 721 tissues in each metacell respectively. Heatmaps show the confusion matrix (the pairwise 722 similarities between metacells) for CD8 (C) and CD4 (D) metacells. The annotations of 723 different metacell groups are shown on the right. E, F. 2D projections of the composition of 724 CD8 (E) and CD4 (F) T cells from different clusters. P, peripheral blood; N, adjacent normal 725 tissue; $\mathrm{T}$, tumor tissue.

726 Figure 5 The correlation and expression analyses of signature IncRNAs associated with 727 different $\mathbf{T}$ cell states

728 A, B. Gene-gene correlation heatmap for signature lncRNA and anchor genes in CD8 (A) and 729 CD4 (B) T cells. The signature gene modules and two anchor genes (CTLA4 and FOXP3) are 730 labelled on the right. C. Expression of signature lncRNA and anchor genes across CD8 731 metacells. Metacells and metacell groups associated with effector and exhausted functions are 732 shown on the bottom. The anchor genes are marked with red color on the right.

\section{Figure 6 Functional annotation analyses of signature IncRNAs}

734 A-C. Functional enrichment maps of CD8 effector/exhausted (A), CD4 effector/exhausted (B) 735 and CD4 Treg (C) signature lncRNAs. The enriched gene sets from Gene Ontology based on 736 the predicted functions of signature lncRNA genes are visualized by Cytoscape plugin 737 Enrichment Map. Each node represents a gene set; size of the node is indicative of the 
738 number of genes and the color intensity reflects the level of significance. Effector signature

739 gene sets are shown in red circles, exhausted or Treg ones in green and the common gene sets

740 in orange. Maps are differently magnified for easier visualization. D-F. The genomic view

741 (D), co-expressed genes (E) and functional annotations (F) of effector signature lncRNA

742 TM4SF19-AS1. G-I. The genomic view (G), co-expressed genes (H) and functional 743 annotations (I) of exhausted signature lncRNA XLOC-633950 (novel). The genomic views

744 are generated from UCSC genome browser. In $(\mathbf{E})$ and $(\mathbf{H})$, co-expressed protein-coding, 745 reference lncRNA and novel lncRNA genes are colored by pink, light green and light yellow 746 respectively.

\section{Supplementary material}

\section{Figure S1 The statistics of $\mathbf{T}$ cell data analysis}

749 A. The number of cells in different subsets across all patients from three cancer types. B, C. 750 The number (B) and the ratio (C) of uniquely mapped read pairs of T cell sequencing data. $\mathbf{D}$.

751 The number of splices of mapping results. E. The different strategies used to explore the best 752 way to obtain novel transcripts. F. The number of assembled transcripts in each subset. PTC, $753 \mathrm{CD}^{+}$cytotoxic $\mathrm{T}$ cells from peripheral blood; TTC, $\mathrm{CD} 8^{+}$cytotoxic $\mathrm{T}$ cells from tumor 754 tissue; NTC, $\mathrm{CD}^{+}$cytotoxic T cells from adjacent normal tissue; $\mathrm{PTH}, \mathrm{CD} 4^{+} \mathrm{CD} 25^{-}$cells 755 from peripheral blood; TTH, CD $4^{+} \mathrm{CD} 25^{-}$cells from tumor tissue; NTH, CD $4^{+} \mathrm{CD} 25^{-}$cells 756 from adjacent normal tissue; PTR, $\mathrm{CD} 4^{+} \mathrm{CD} 25^{\mathrm{hi}}$ cells from peripheral blood; TTR, $757 \mathrm{CD}^{+} \mathrm{CD} 25^{\text {hi }}$ cells from tumor tissue; NTR, CD4 ${ }^{+} \mathrm{CD} 25^{\text {hi }}$ cells from adjacent normal tissue; 758 PTY, $\mathrm{CD} 4{ }^{+} \mathrm{CD} 25^{\text {int }}$ cells from peripheral blood; TTY, $\mathrm{CD} 4{ }^{+} \mathrm{CD} 25^{\text {int }}$ cells from tumor tissue; $759 \mathrm{NTY}, \mathrm{CD}^{+} \mathrm{CD} 25^{\text {int }}$ cells from adjacent normal tissue; PPQ, CD4 ${ }^{+} \mathrm{T}$ cells from peripheral 760 blood; TPQ, CD4 ${ }^{+} \mathrm{T}$ cells from tumor tissue; NPQ, $\mathrm{CD}^{+} \mathrm{T}$ cells from adjacent normal tissue; 761 CRC, colorectal cancer; HCC, hepatocellular carcinoma; NSCLC, non-small-cell lung cancer.

762 Figure S2 The cluster hierarchy of metacells

763 A, B. The cluster hierarchy of CD8 (A) and CD4 (B) metacells. Subtrees in blue, sibling 764 subtrees in gray. The metacells are colored and labelled on bottom.

765 Figure S3 2D projections of CD8 T cells 
766 A, B. The composition of CD8 T cells from different clusters (A) and cancer types (B).

767 Metacells and the cells involved in them are marked by different colors. The number of cells

768 within each cluster is shown in brackets.

$769 \quad$ Figure S4 2D projections of CD4 T cells

770 A, B. The composition of CD4 T cells from different clusters (A) and cancer types (B).

771 Metacells and the cells involved in them are marked by different colors. The number of cells

772 within each cluster is shown in brackets.

773 Figure S5 Expression of signature IncRNA and anchor genes across CD4 metacells

774 Metacells and metacell groups associated with effector, exhausted and Treg functions are

775 shown on the bottom. The anchor genes are marked with red color on the right.

776 Figure S6 Functional enrichment maps of shared signature IncRNAs

777 A-C. Functional enrichment maps of shared signature lncRNAs between CD8 effector and

778 CD4 effector function (A), between CD8 exhausted and CD4 exhausted function (B) and

779 between CD4 exhausted and CD4 Treg function $(\mathbf{C})$. Each node represents a gene set; size of

780 the node is indicative of the number of genes and the color intensity reflects the level of

781 significance. Maps are differently magnified for easier visualization.

782 Table S1 The basic information of single $\mathbf{T}$ cell data

783 Table S2 Clinical characteristics of three cancer patients

784 Table S3 The list of novel lncRNAs successfully validated by Sanger sequencing

785 Table S4 The list of specific-expressed IncRNAs

786 Table S5 The composition of CD8 metacells

787 Table S6 The composition of CD4 metacells

$788 \quad$ Table S7 The list of signature IncRNAs

789 Table S8 Functional annotations of 84 signature IncRNAs

790 Table S9 Functional enrichment results of CD8 effector/exhausted signature IncRNAs

791 Table S10 Functional enrichment results of CD4 effector/exhausted signature IncRNAs 
bioRxiv preprint doi: https://doi.org/10.1101/2020.07.22.215855; this version posted July 24, 2020. The copyright holder for this preprint (which was not certified by peer review) is the author/funder. All rights reserved. No reuse allowed without permission.

792 Table S11 Functional enrichment results of CD4 Treg signature IncRNAs

793 Supplementary Table1-8 are Excel format, and Supplementary Table9-11 are Word format. 
bioRxiv preprint doi: https://doi.org/10.1101/2020.07.22.215855; this version posted July 24, 2020. The copyright holder for this preprint (which was not certified by peer review) is the author/funder. All rights reserved. No reuse allowed without permission.

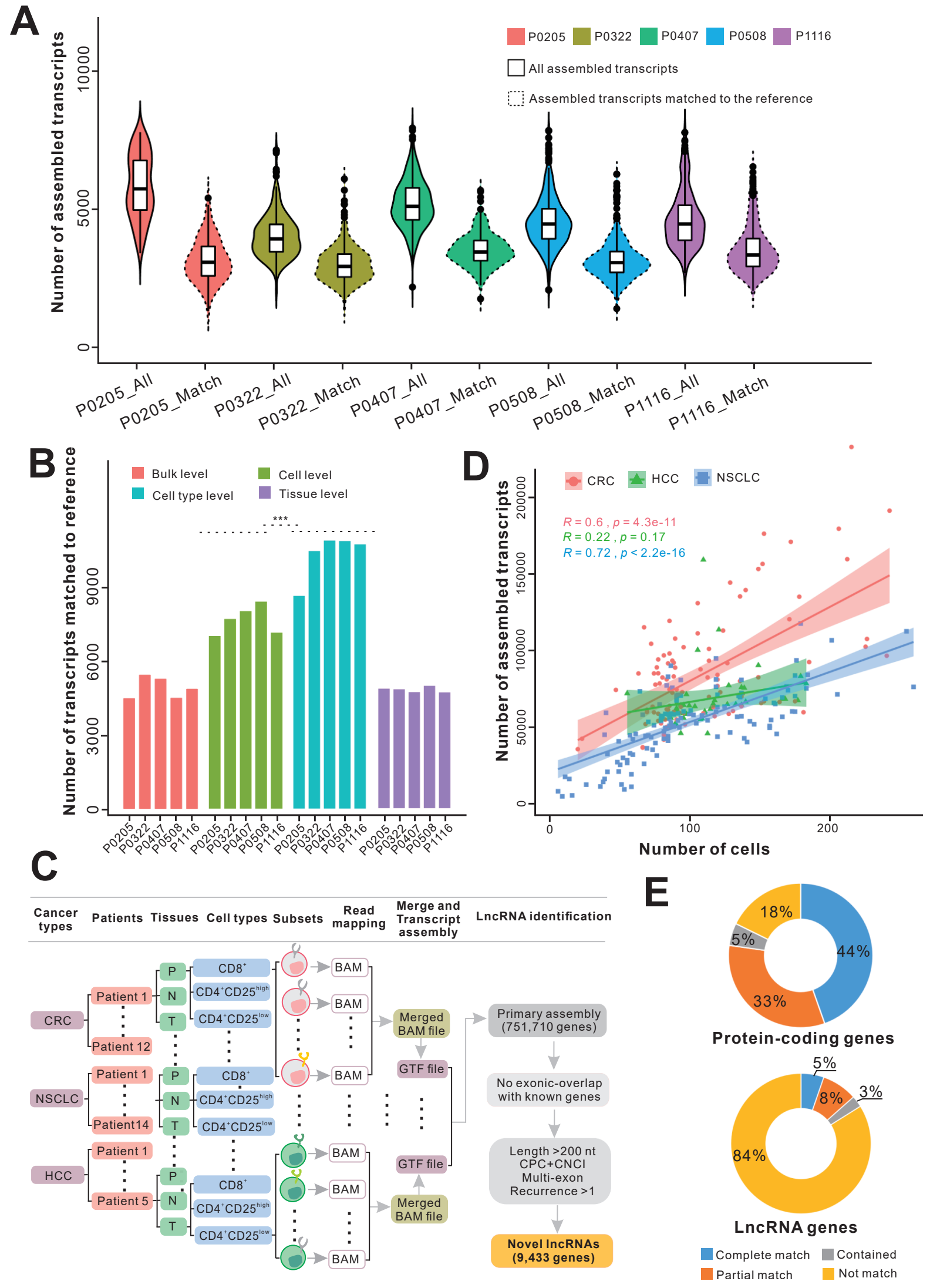


bioRxiv preprint doi: https://doi.org/10.1101/2020.07.22.215855; this version posted July 24, 2020. The copyright holder for this preprint (which was not certified by peer review) is the author/funder. All rights reserved. No reuse allowed without permission.

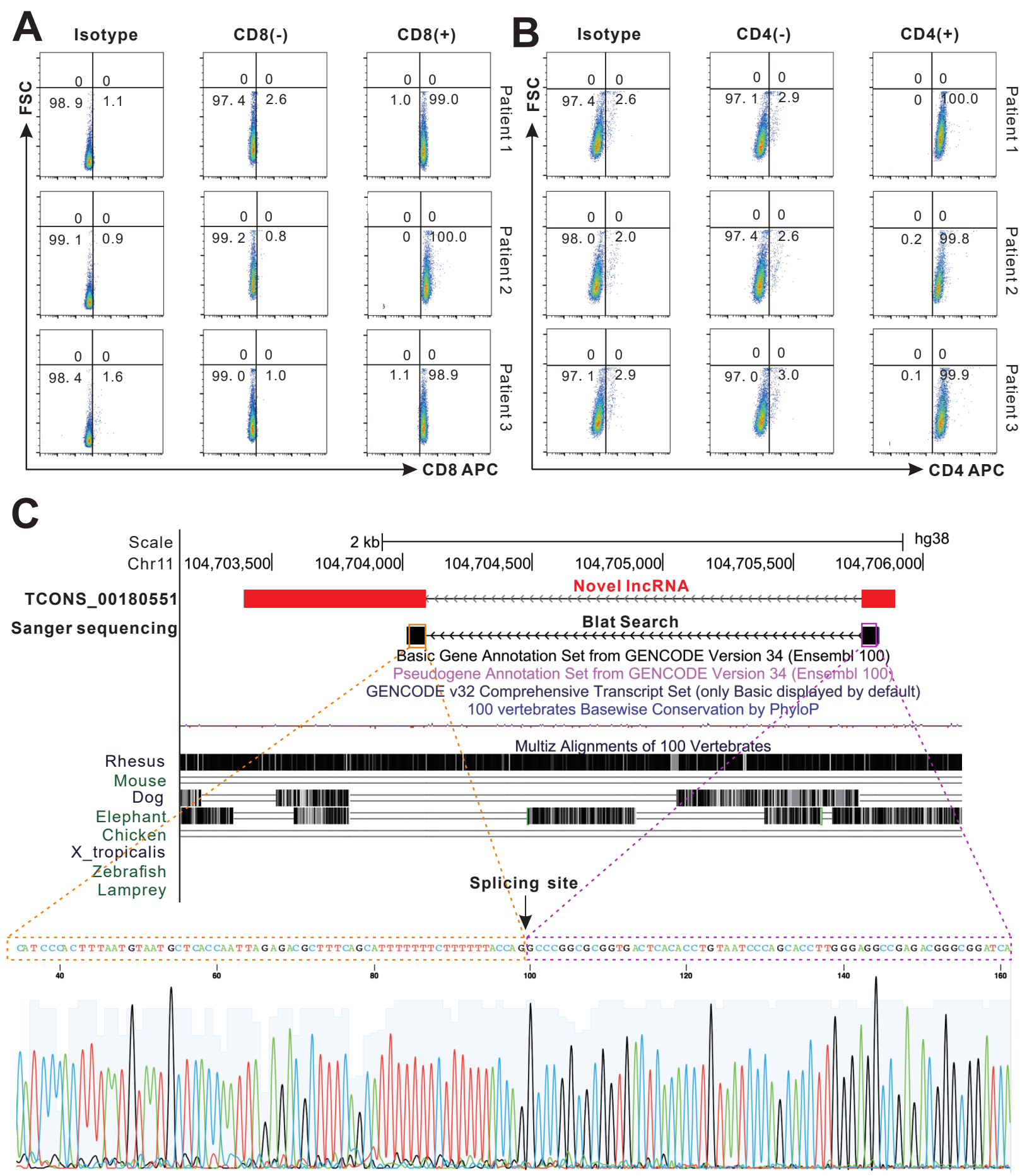


bioRxiv preprint doi: https://doi.org/10.1101/2020.07.22.215855; this version posted July 24, 2020. The copyright holder for this preprint (which was not certified by peer review) is the author/funder. All rights reserved. No reuse allowed without permission.

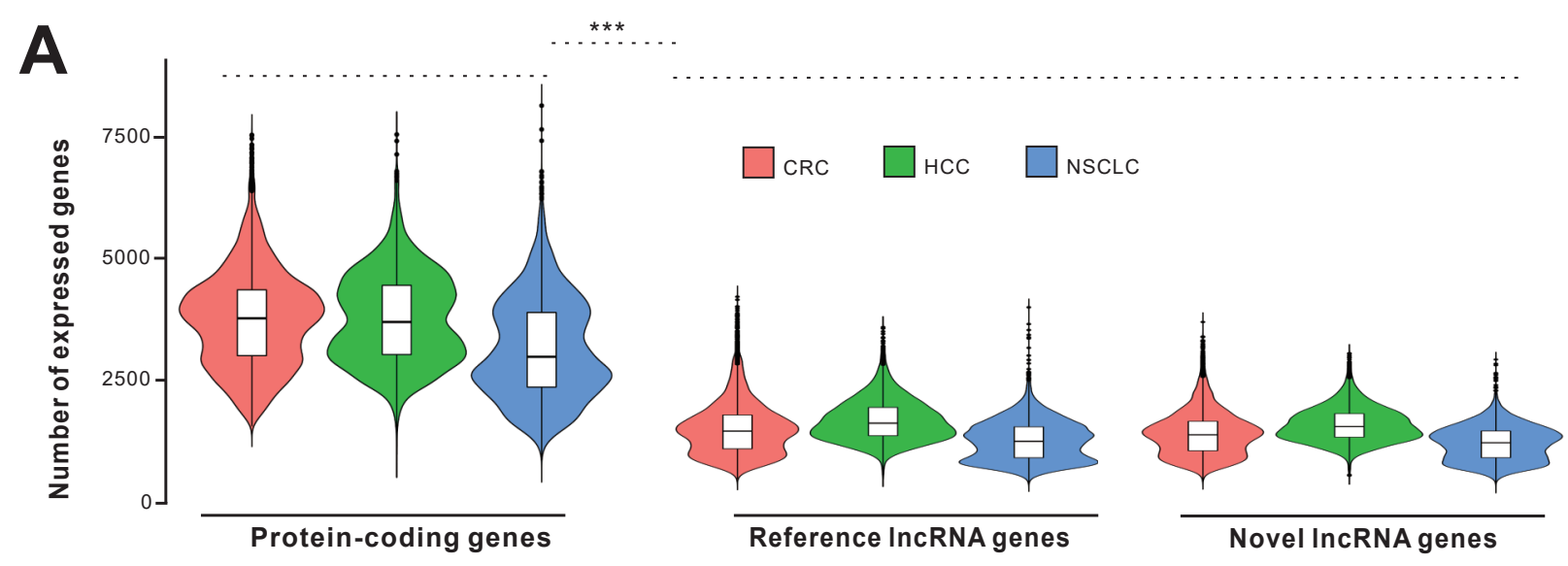

B
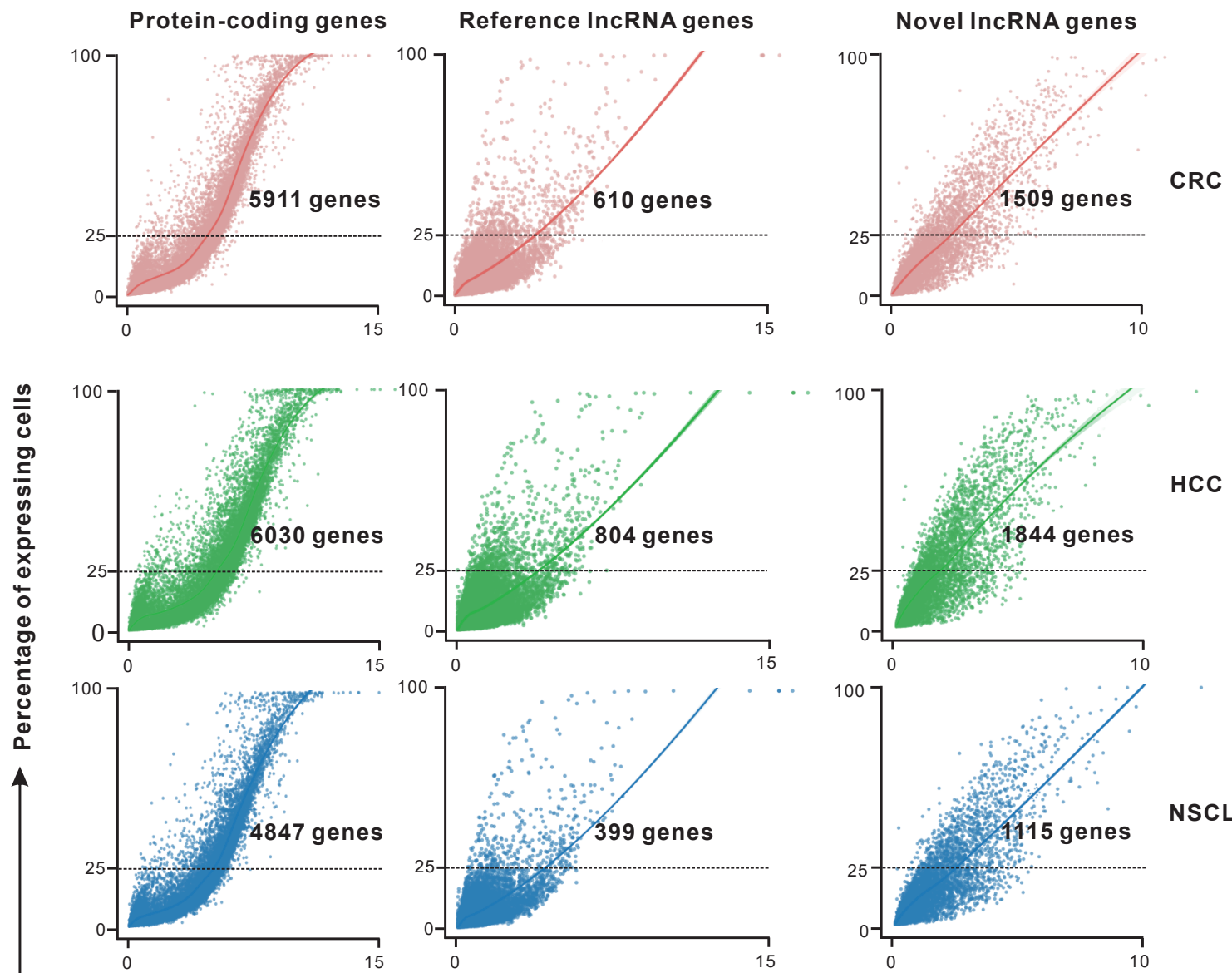

HCC

NSCLC

Mean expression 
bioRxiv preprint doi: https://doi.org/10.1101/2020.07.22.215855; this version posted July 24, 2020. The copyright holder for this preprint (which was not certified by peer review) is the author/funder. All rights reserved. No reuse allowed without permission.
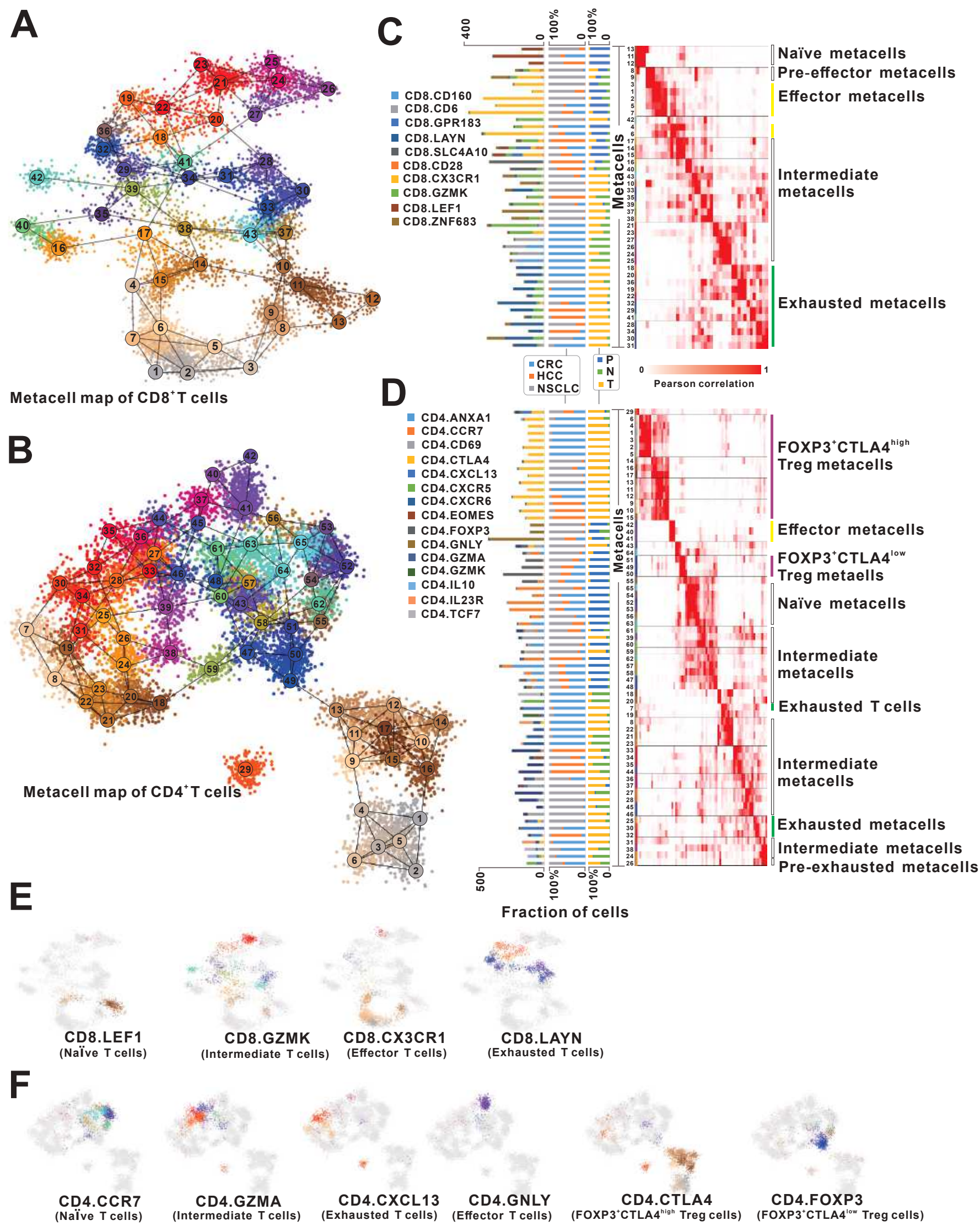

Fraction of cells
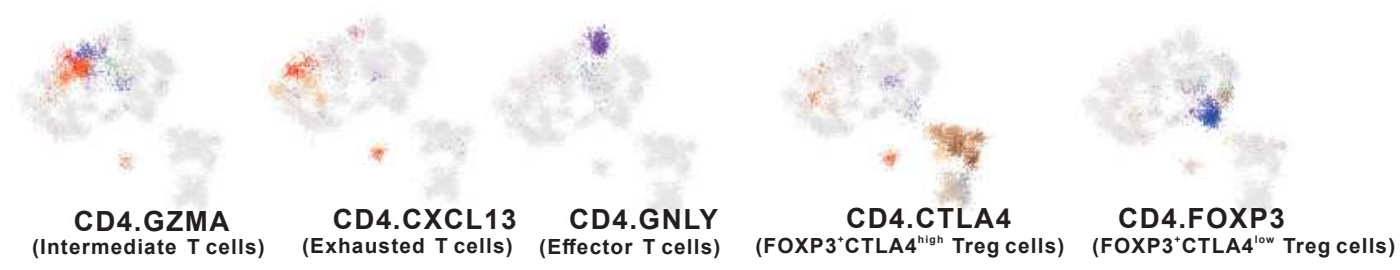
bioRxiv preprint doi: https://doi.org/10.1101/2020.07.22.215855; this version posted July 24, 2020. The copyright holder for this preprint (which was not certified by peer review) is the author/funder. All rights reserved. No reuse allowed without permission.

A

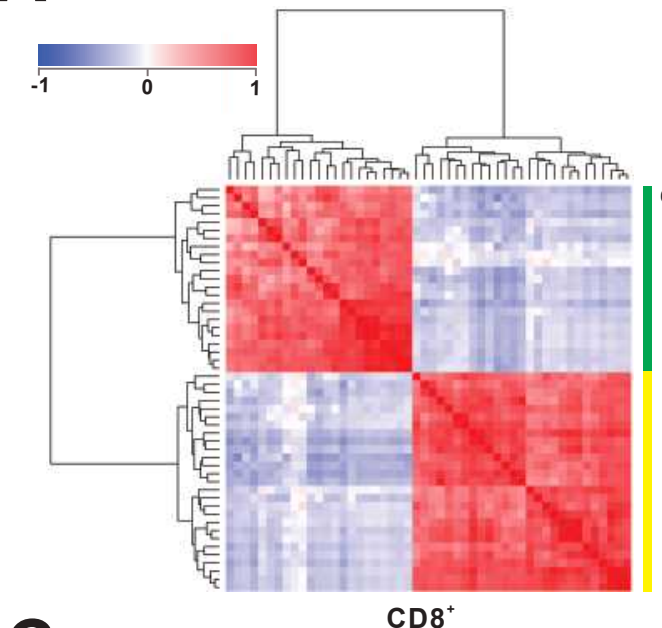

C

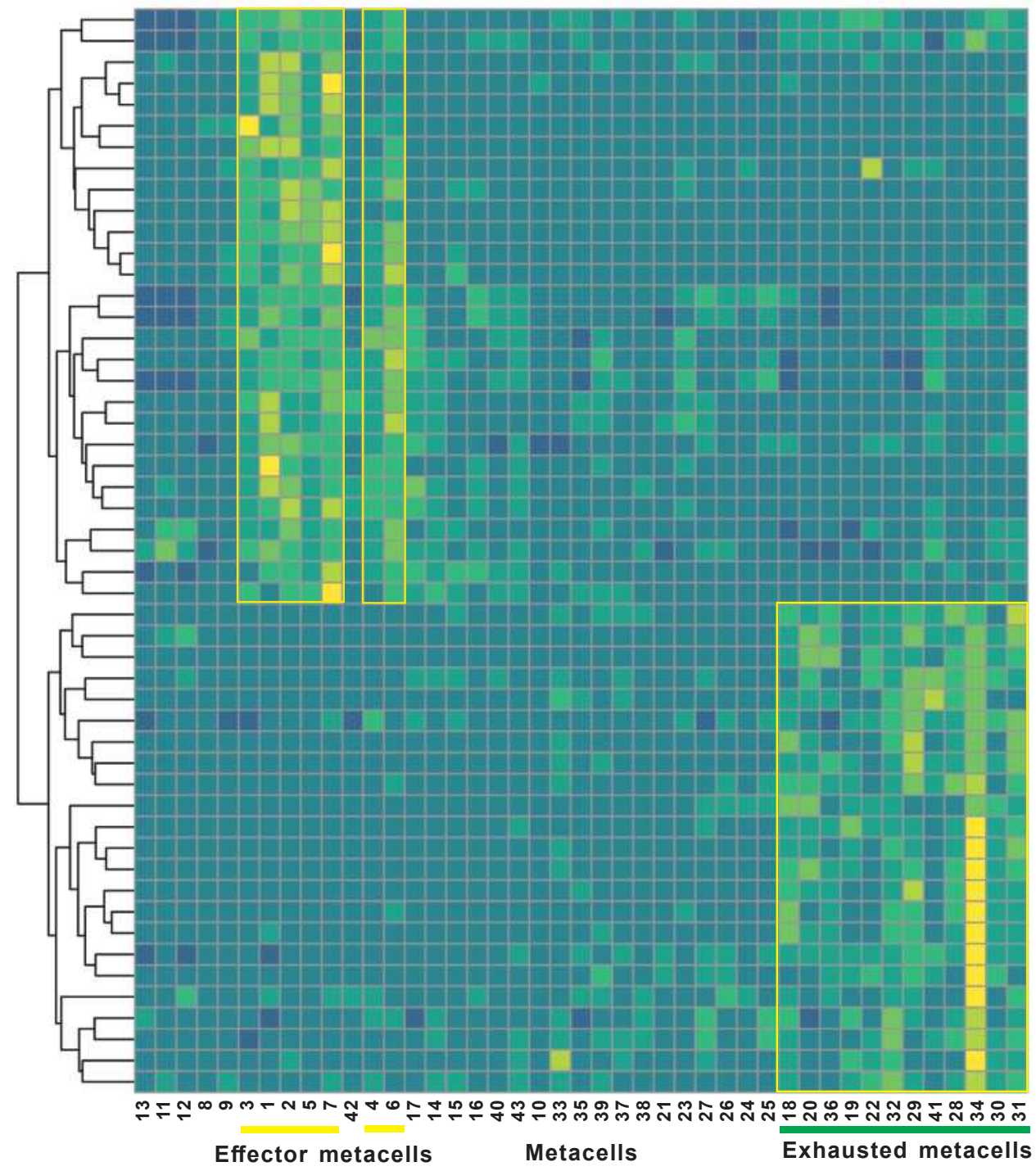

Effector metacells

Metacells

Exhausted metacells

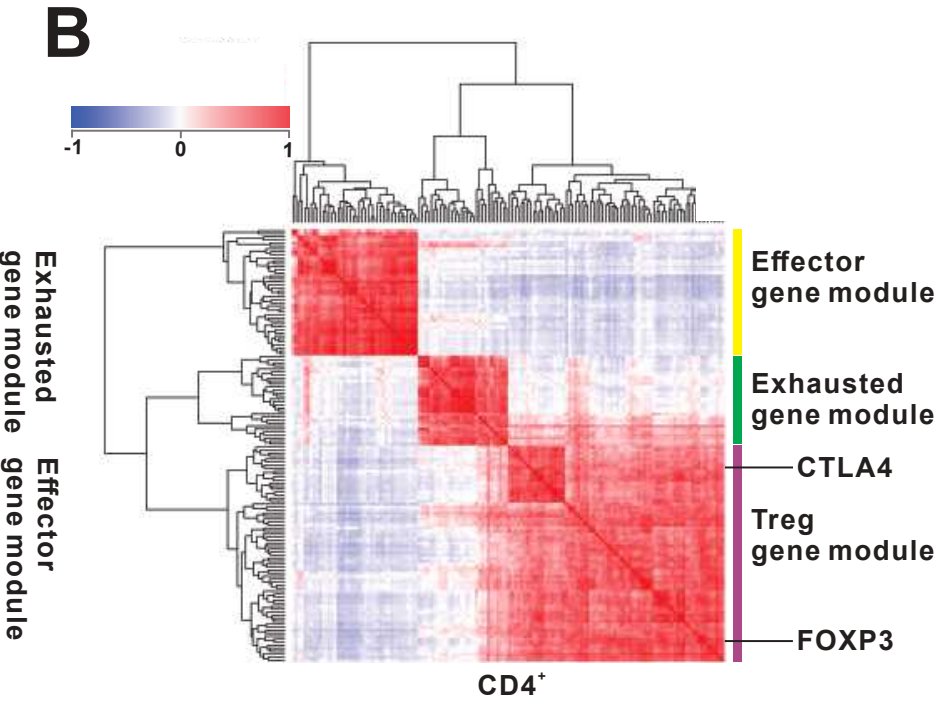

GZMH

PRF1

TM4SF19-AS1

MSTRG.34555

XLOC-593046

MSTRG. 25773

LOC101927369

LOC101927441

LOC105376876

FGFBP2

CX3CR1

MSTRG.43215

LOC102724104

MSTRG.59493

XLOC-591096

XLOC-501240

MSTRG.16157

LINC00943

XLOC-503860

XLOC-501334

XLOC-160463

AC009495.2

LOC102725160

XLOC-503759

MCM 3 AP-AS 1

LINC01278

RP11-539L10.2

XLOC-188938

MSTRG.69890

XLOC-501348

CTLA4

RP11-440D17.3

LOC105369905

NRAV

RP11-146I2.1

AC069363.1

TIGIT

XLOC-304931

LAG3

MIR155HG

HAVCR2

PDCD1

LOC100130093

LOC105378513

RP11-139H15.5

IL21-AS1

RP11-181G12.2

LOC101928710

CTD-2357A8.3

CTA-384D8.35

RP5-1074L1.4

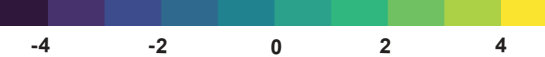


bioRxiv preprint doi: https://doi.org/10.1101/2020.07.22.215855; this version posted July 24, 2020. The copyright holder for this preprint (which was not certified by peer review) is the author/funder. All rights reserved. No reuse allowed without permission.
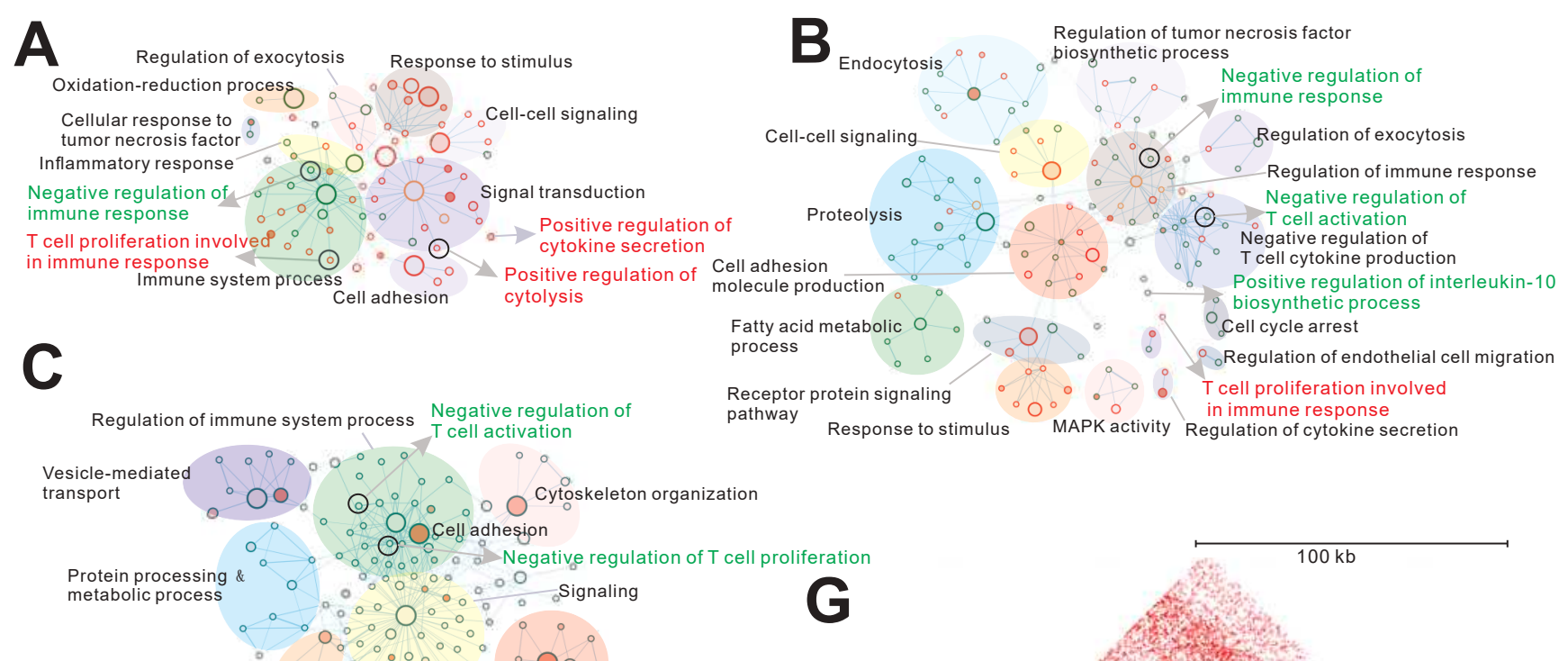

Regulation of cell migrations, 000000

Cell cycle arrest Cellular lipid metabolic process

D
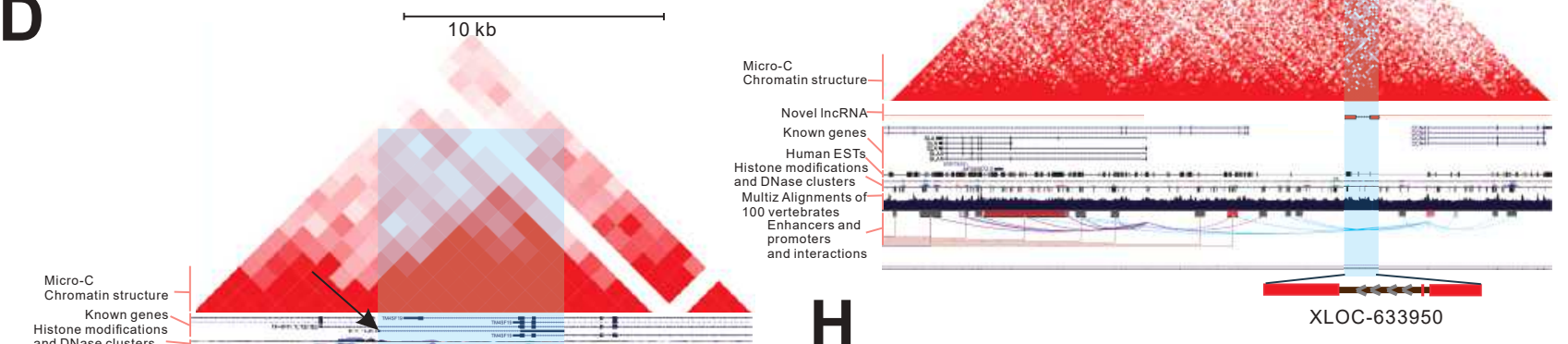

and Nase

ancers and promoters.

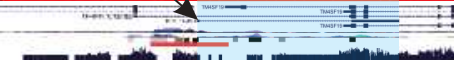

Multiz Alignments of

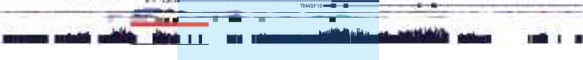

TM4SF19-AS1

$\mathbf{E}$
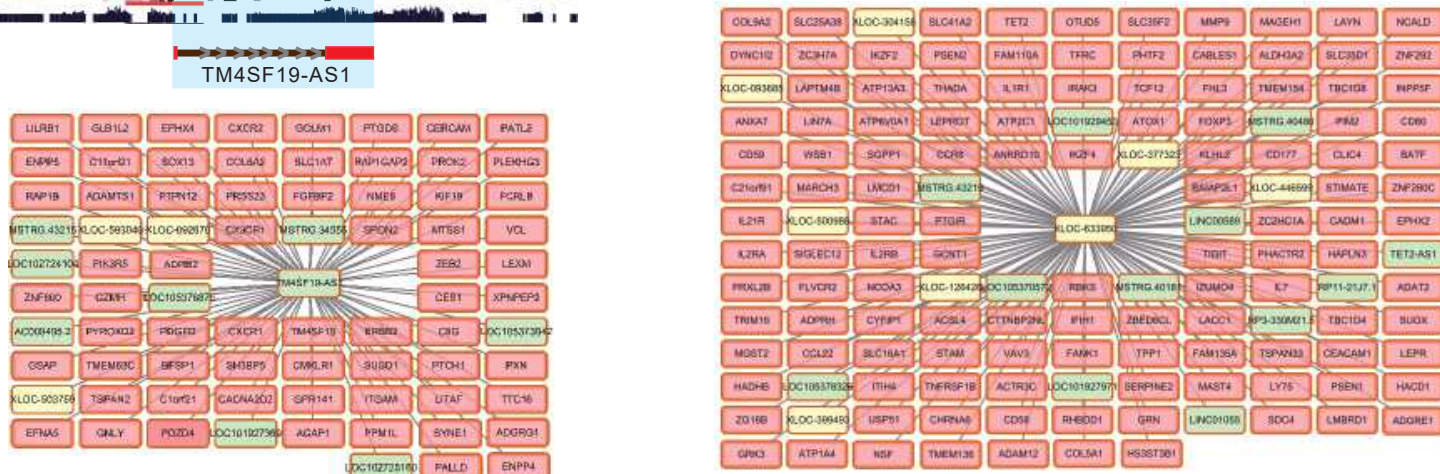

$\mathbf{F}$

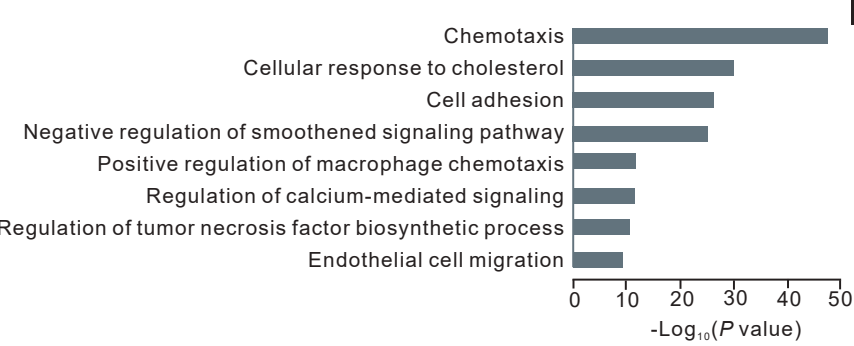

Negative regulation of interleukin-2 production

Negative regulation of $\mathrm{T}$ cell cytokine production Negative regulation of interferon-gamma production Negative regulation of $\mathrm{T}$ cell proliferation Negative regulation of $\mathrm{T}$ cell activation Negative regulation of histone deacetylation Regulation of regulatory $T$ cell differentiation Negative regulation of immune respons

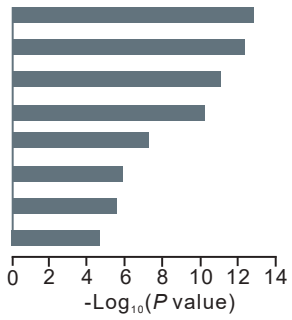

